\title{
Alteration of Platelet Count in Patients with Severe Non-Plasmodium falciparum Malaria: A Systematic Review and Meta-Analysis
}

\author{
Aongart Mahittikorn ${ }^{1}$, Frederick Ramirez Masangkay ${ }^{2}$, Kwuntida Uthaisar Kotepui ${ }^{3}$, Wanida Mala ${ }^{3}$, \\ Giovanni De Jesus Milanez ${ }^{4} \mathbb{D}$, Polrat Wilairatana ${ }^{5}$ (D) and Manas Kotepui ${ }^{3, *(\mathbb{D}}$
}

Citation: Mahittikorn, A.; Masangkay, F.R.; Kotepui, K.U.; Mala, W.; Milanez, G.D.J.; Wilairatana, P.; Kotepui, M. Alteration of Platelet Count in Patients with Severe Non-Plasmodium falciparum Malaria: A Systematic Review and Meta-Analysis. Biology 2021, 10, 1275. https://doi.org/10.3390/ biology10121275

Academic Editor: Michal Tomasz Marzec

Received: 26 October 2021 Accepted: 3 December 2021 Published: 5 December 2021

Publisher's Note: MDPI stays neutral with regard to jurisdictional claims in published maps and institutional affiliations.

Copyright: (c) 2021 by the authors. Licensee MDPI, Basel, Switzerland. This article is an open access article distributed under the terms and conditions of the Creative Commons Attribution (CC BY) license (https:/ / creativecommons.org/licenses/by/ $4.0 /)$.
1 Department of Protozoology, Faculty of Tropical Medicine, Mahidol University, Bangkok 10400, Thailand; aongart.mah@mahidol.ac.th

2 Department of Medical Technology, Institute of Arts and Sciences, Far Eastern University-Manila, Manila 1008, Philippines; frederick_masangkay2002@yahoo.com

3 Medical Technology, School of Allied Health Sciences, Walailak University, Tha Sala, Nakhon Si Thammarat 80160, Thailand; kwuntida.ut@wu.ac.th (K.U.K.); wanida.ma@wu.ac.th (W.M.)

4 Department of Medical Technology, Faculty of Pharmacy, University of Santo Tomas, Manila 1008, Philippines; Gmilanez81@gmail.com

5 Department of Clinical Tropical Medicine, Faculty of Tropical Medicine, Mahidol University, Bangkok 10400, Thailand; polrat.wil@mahidol.ac.th

* Correspondence: manas.ko@wu.ac.th; Tel.: +66-9-5439-2469

Simple Summary: Alteration of platelet count is frequently seen in patients with malaria. However, the platelet biology under severe pathological conditions like severe malaria remains unclear. The present systematic review collated literatures and synthesized the evidence regarding the risks of severe and profound thrombocytopenia in patients with severe non-Plasmodium falciparum malaria. We found that the high prevalence of severe and profound thrombocytopenia in patients with severe non-P. falciparum malaria at $47 \%$ and $20 \%$, respectively. Moreover, deaths were seen in patients with severe and profound thrombocytopenia at $11 \%$. Therefore, the present systematic review provided the new insight into the clinical relevance of severe and profound thrombocytopenia in diagnosing and managing non-P. falciparum malaria. Severe thrombocytopenia should serve as a warning sign of increased chances of complications. Meanwhile, profound thrombocytopenia should serve as a marker of mortality among patients with non-P. falciparum malaria.

Abstract: The understanding of platelet biology under physiological and pathological conditions like malaria infection is critical importance in the context of the disease outcome or model systems used. The importance of severe thrombocytopenia (platelet count $<50,000$ cells $(\mu \mathrm{L})$ and profound thrombocytopenia (platelet count $<20,000$ cells $/ \mu \mathrm{L}$ ) in malaria patients remains unclear. This study aimed to synthesize evidence regarding the risks of severe and profound thrombocytopenia in patients with severe non-Plasmodium falciparum malaria. Our overall aim was to identify potential indicators of severe non-P. falciparum malaria and the Plasmodium species that cause severe outcomes. This systematic review was registered at the International Prospective Register of Systematic Reviews (PROSPERO) under registration ID CRD42020196541. Studies were identified from previous systematic reviews $(n=5)$ and the MEDLINE, Scopus, and Web of Science databases from 9 June 2019 to 9 June 2020. Studies were included if they reported the outcome of severe non-Plasmodium species infection, as defined by the World Health Organization (WHO) criteria, in patients with known platelet counts and/or severe and profound thrombocytopenia. The risk of bias was assessed using the Newcastle-Ottawa Scale (NOS). Data were pooled, and pooled prevalence (PP) and pooled odds ratios (ORs) were calculated using random effects models. Of the 118 studies identified from previous meta-nalyses, 21 met the inclusion criteria. Of the 4807 studies identified from the databases, three met the inclusion criteria. Nine studies identified from reference lists and other sources also met the inclusion criteria. The results of 33 studies reporting the outcomes of patients with severe P. vivax and P. knowlesi infection were pooled for meta-analysis. The PP of severe thrombocytopenia (reported in 21 studies) was estimated at 47\% (95\% confidence interval (CI): 33-61\%, $\mathrm{I}^{2}$ : 96.5\%), 
while that of profound thrombocytopenia (reported in 13 studies) was estimated at $20 \%$ (95\% CI: $14-27 \%, 85.2 \%)$. The pooled weighted mean difference (WMD) in platelet counts between severe uncomplicated Plasmodium infections (reported in 11 studies) was estimated at $-28.51 \%(95 \% \mathrm{CI}$ : $-40.35-61 \%, \mathrm{I}^{2}: 97.7 \%$ ), while the pooled WMD in platelet counts between severe non-Plasmodium and severe $P$. falciparum infections (reported in eight studies) was estimated at $-3.83 \%$ (95\% CI: $-13.90-6.25 \%, \mathrm{I}^{2}: 85.2 \%$ ). The pooled OR for severe/profound thrombocytopenia comparing severe to uncomplicated Plasmodium infection was 2.92 (95\% CI: 2.24-3.81, I²: 39.9\%). The PP of death from severe and profound thrombocytopenia was estimated at $11 \%$ (95\% CI: $0-22 \%)$. These results suggest that individuals with severe non-P. falciparum infection (particularly P. vivax and P. knowlesi) who exhibit severe or profound thrombocytopenia should be regarded as high risk, and should be treated for severe malaria according to current WHO guidelines. In addition, severe or profound thrombocytopenia coupled with other clinical and microscopic parameters can significantly improve malaria diagnosis, enhance the timely treatment of malaria infections, and reduce the morbidity and mortality of severe non-P. falciparum malaria.

Keywords: platelet; thrombocytopenia; falciparum; malaria

\section{Introduction}

An understanding of platelet biology under physiological and pathological conditions like malaria infection is of critical importance in the context of the disease outcome or model systems used. Platelets are produced from megakaryocytes and play a crucial role in hemostasis and thrombus formation [1]. Thrombocytopenia (platelet count $<100,000$ cells $/ \mu \mathrm{L}$ ) is common in patients with malaria [2-4]. In patients with Plasmodium falciparum and Plasmodium vivax infections, thrombocytopenia is associated with increased mortality $[5,6]$. The proposed pathogenic mechanisms during $P$. falciparum malaria are increased platelet activation and consumption by factors such as the cytoadherence of infected erythrocytes and subsequent endothelial damage [7], platelet accumulation in the microvasculature [8], bone marrow alterations, and antibody-mediated platelet destruction [9]. Severe thrombocytopenia is defined as a platelet count of $<50,000$ cells $/ \mu \mathrm{L}$, while profound thrombocytopenia is defined as a platelet count of $<20,000$ cells $/ \mu \mathrm{L}$ [10]. Severe or profound thrombocytopenia has been reported in patients with $P$. falciparum and $P$. vivax malaria and is associated with bleeding [11] and disseminated intravascular coagulation [12].

Severe malaria is defined as the presence of Plasmodium parasitemia and one or more of the following manifestations: impaired consciousness, prostration, acidosis, hypoglycemia, severe malarial anemia, renal impairment, jaundice, pulmonary edema, significant bleeding, shock, or hyperparasitemia [13]. Severe or profound thrombocytopenia has been widely documented in patients with severe P. falciparum malaria, although it is not included in the current World Health Organization (WHO) criteria for severe P. falciparum malaria [13]. A previous study reported that adults with P. falciparum malaria and profound thrombocytopenia were five times more likely to die than patients with higher platelet counts [14]. Although severe thrombocytopenia has been widely recognized as a feature of patients with severe P. falciparum infection, information on the prevalence of severe or profound thrombocytopenia in patients with non-P. falciparum malaria (including P. vivax, P. malariae, P. ovale, and P. knowlesi infection) is limited. The most recent systematic review on severe thrombocytopenia in $P$. vivax malaria demonstrated that severe thrombocytopenia was equally common in P. vivax and P. falciparum malaria [15]. However, the results were interpreted based on a small number of studies with low sample sizes, and the estimates had wide confidence intervals. In addition, the study did not report the outcomes of severe $P$. vivax infection. To clarify this issue, we conducted a meta-analysis of studies reporting severe or profound thrombocytopenia in patients with severe $P$. vivax, P. malariae, P. ovale, and P. knowlesi malaria. Our goal was to assess whether severe or profound thrombocytopenia could inform clinical decision-making, and whether the degree 
of thrombocytopenia had predictive utility independent of current clinical and laboratory prognostic indices.

\section{Methods}

\subsection{Study Registration}

This systematic review was registered at the International Prospective Register of Systematic Reviews (PROSPERO) under registration ID CRD42020196541.

\subsection{Types of Studies and Inclusion and Exclusion Criteria}

Original studies were included if they were observational, prospective, or retrospective in design, and reported outcomes of patients with: (1) severe malaria as defined by the WHO (impaired consciousness, jaundice, pulmonary edema, acute renal failure, severe anemia, bleeding, acidosis, hyperparasitemia, shock, respiratory distress, or hypoglycemia); (2) non-Plasmodium falciparum including $P$. vivax, $P$. malariae, $P$. ovale, and $P$. knowlesi infections; (3) severe thrombocytopenia (platelet count $<50,000$ cells $/ \mu \mathrm{L}$ ) or profound thrombocytopenia (platelet count $<20,000$ cells $/ \mu \mathrm{L}$ ); (4) severe and uncomplicated malaria with known platelet counts; or (5) severe P. falciparum and non- $P$. falciparum infections with known platelet counts. The following types of studies were excluded: (1) studies examining severe non-Plasmodium infection co-morbidity with other diseases, (2) studies conducted among pregnant patients, and (3) studies in which platelet counts could not be extracted. The systematic review was performed following the Preferred Reporting Items for Systematic Reviews and Meta-Analyses (PRISMA) guidelines (see PRISMA Checklist S1).

\subsection{Search Strategy and Data Extraction}

Potentially relevant studies were identified from three sources. The first source of studies was previous systematic reviews $(n=5)$ reporting outcomes of severe $P$. vivax, P. malariae, P. ovale, and P. knowlesi infections. The second source of studies was a systematic search of relevant databases (MEDLINE, Scopus, and ISI Web of Science) between 1 June 2020 and 9 June 2020 using MeSH headings (Severe OR complicated OR complication) AND (malaria OR plasmodium) AND (thrombocytopenia OR "low platelet"), with no restrictions on language. The third source of studies was the reference lists of the included studies as well as other sources (e.g., Google Scholar). Two authors (AM and MK) independently screened the titles and abstracts. Any disagreements in full-text article selection were resolved by consensus. Potentially relevant full-text articles were obtained, read, and extracted by two independent authors concerning the inclusion criteria. Data were extracted into a standardized form using an Excel spreadsheet. For all studies, data on authors, year of publication, study location, year of publication, and study design were extracted. In addition, data on mean/median age of participants, male percentage, mean/median platelet count, parasitemia level, number of patients with severe and profound thrombocytopenia, Plasmodium species, number of patients with severe malaria, number of patients with severe or profound thrombocytopenia, and number of deaths were extracted. Data extraction was undertaken by one author and checked by all authors.

\subsection{Assessment of Risk of Bias}

The risk of bias was independently assessed by two authors (A.M. and M.K.). As the included studies were observational retrospective, prospective, and case-control studies, the Newcastle-Ottawa Scale (NOS) tool was used [16]. The NOS tool allows for quality assessment in three domains, including the selection of study groups, comparability of groups, and ascertainment of the outcome. The NOS used as the primary outcome in this meta-analysis was the pooled prevalence (PP) of severe or profound thrombocytopenia among one group (patients with severe non-P. falciparum malaria) and not the comparator groups. Therefore, only three domains were used to assess the included studies: case definition, representativeness of cases, and ascertainment of the outcome. The risk of bias 
was reported as low (1 star), moderate ( 2 stars), or high ( 3 stars) based on the total of the three scores retrieved from each domain. Any discrepancies in ratings were addressed in the discussion.

\subsection{Data Synthesis and Analysis}

The following three outcomes were analyzed by meta-analysis: (1) PP and 95\% confidence interval (CI) of severe and profound thrombocytopenia in individuals with severe non-P. falciparum infection, estimated using the random-effects DerSimonian and Laird models [17]; (2) pooled odds ratios (ORs) in individuals with severe non- $P$. falciparum infection compared with individuals with uncomplicated non-P. falciparum infection, estimated using fixed- or random-effects models depending on the level of heterogeneity; (3) pooled ORs in individuals with severe non- $P$. falciparum infection compared with individuals with severe $P$. falciparum infection, estimated using fixed- or random-effects models depending on the level of heterogeneity. The heterogeneity and level of heterogeneity of studies were assessed using Cochran's Q and ${ }^{2}$ (inconsistency) statistics, respectively. Publication bias was evaluated using visual inspection of funnel plots and Egger's tests. If any small-study effects were observed, a further contour-enhanced funnel plot was generated to assess if asymmetry resulted from publication bias or other factors. The meta-analysis was carried out using Stata software. To explore whether a single study affected the heterogeneity or the conclusions of the meta-analysis, sensitivity analyses omitting each study were performed in turn. Subgroup analyses were conducted to evaluate potential sources of heterogeneity.

\section{Results}

Twenty-one studies reporting the outcomes of severe non-P.falciparum malaria were retrieved from previous systematic reviews and meta-analyses [18-22]. As previous systematic reviews included studies dating until 2019, additional searches between 2019 and 2020 were also performed, which identified 4807 unique articles. Of the titles and abstracts screened, 518 articles were selected for full-text screening (Figure 1). Three studies were eligible for inclusion. Additional searches of reference lists and other sources identified nine articles meeting the inclusion criteria. A total of 33 studies were included in this systematic review and assessed for methodological quality $[10,23-54]$.

\subsection{Characteristics of the Included Studies}

Thirty-three studies from Asia (India [10,23,25,31,35-40,43,46,47,49-51], Malaysia [29,30,53,54], Indonesia [32], Papua New Guinea [42], and Republic of Korea [45]) and South America (Brazil [24,33,34,41,48,52] and Colombia [26-28,44]) reported data on 1495 individuals with severe non- $P$. falciparum malaria. The included studies were prospective or retrospective observational studies, case-control studies, and cohort studies. All studies excluded individuals with $P$. falciparum single or mixed infections. Most of the included studies reported the outcomes of severe non-P. falciparum malaria in adults, while Gupta et al., 2016 [36], Lanca et al., 2012 [41], and Singh et al., 2011 [50] reported outcomes of severe non-P. falciparum malaria in children younger than 7 years. Most of the included studies reported outcomes of predominantly male individuals who developed severe non- $P$. falciparum malaria, while Sharma et al., 2012 and Singh et al., 2011 reported a higher proportion of women who developed severe non- $P$. falciparum malaria. All non- $P$. falciparum malaria cases reported in the included studies involved $P$. vivax and $P$. knowlesi malaria. Of the included studies, 30 [10,23-28,30-52] reported outcomes of 1371 cases of severe $P$. vivax single infections, while four $[29,30,53,54]$ reported outcomes of 124 cases with severe $P$. knowlesi infections. Barber et al., 2013 [30] reported outcomes of patients with both severe $P$. vivax and severe P. knowlesi infections. Other characteristics of the included studies are shown in Table S1. 


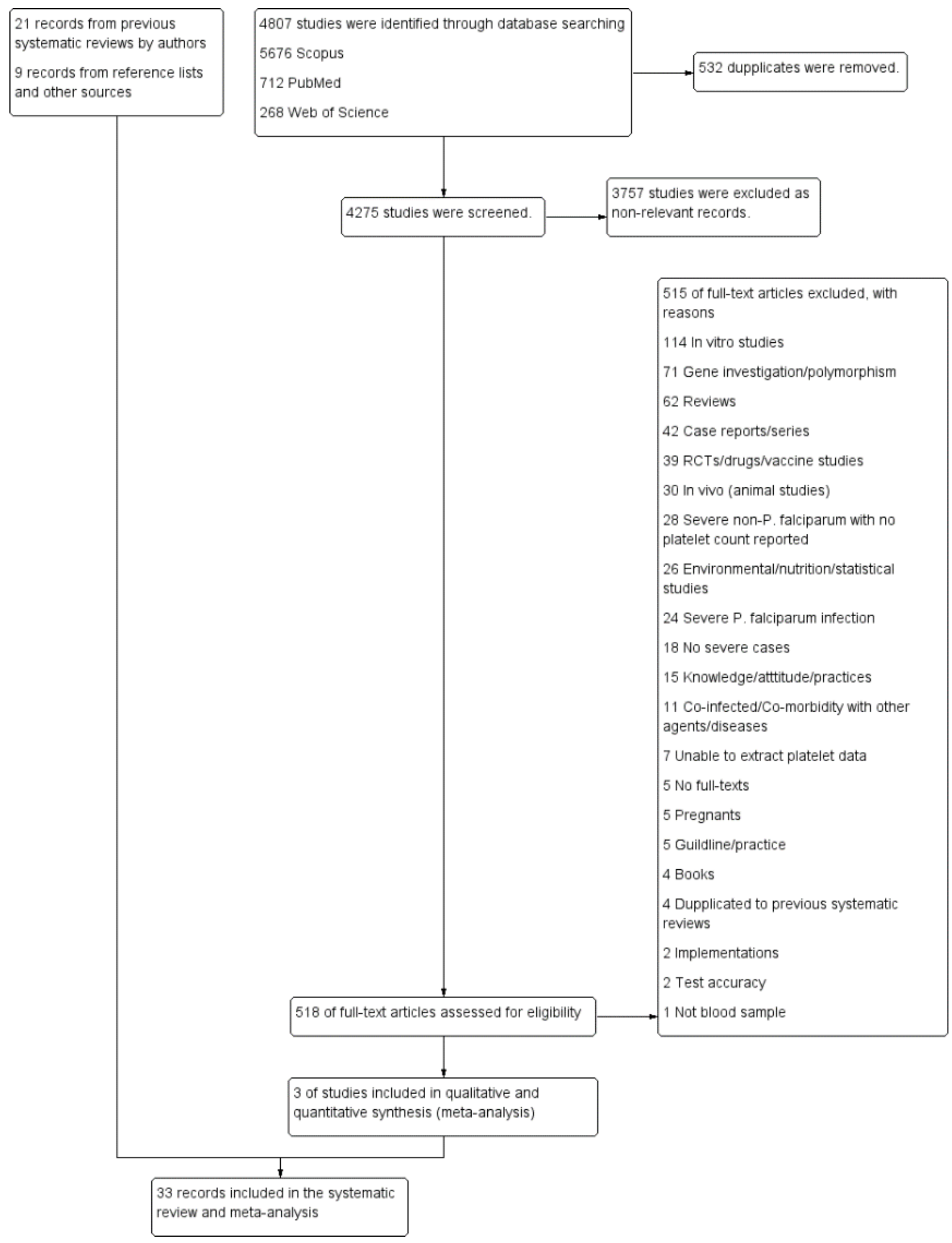

Figure 1. Study flow diagram. The diagram showed the selection process of potentially relevant studies. When duplicates (532 studies) were removed, the remaining studies (4275 studies) were screened for titles and abstracts, and 3757 nonrelevant studies were excluded. Next, 518 studies were examined in full-text, and then 515 studies were excluded for specific reasons. Three studies met the eligibility criteria, and 33 studies retrieved from other sources were included in the meta-analysis.

\subsection{Risk of Bias of Individual Studies}

Of the 33 included studies that were assessed for quality, 28 [10,23-30,32,33,35-48,50-52] were rated as having the highest quality ( 3 stars) while the remaining five studies $[31,33,49,53,54]$ 
were rated as moderate quality ( 2 stars) because of the unclear case selection criteria for inclusion (Table S2).

\subsection{Prevalence of Severe Thrombocytopenia in Severe Non-P. falciparum Malaria}

Data on severe thrombocytopenia ( 349 cases) in patients with severe $P$. vivax malaria (808 cases) from 18 studies [10,24,25,28,32,34,36,37,39-41,43,46-48,50-52] were extracted and pooled in the meta-analysis of proportion. The PP of severe thrombocytopenia in patients with severe $P$. vivax malaria was $42 \%$ (95\% CI: $\left.27-57 \%, \mathrm{I}^{2}: 96.3 \%\right)$. The prevalence reported in individual studies varied highly (range: 3-86\%). Data on severe thrombocytopenia in patients with severe $P$. knowlesi malaria were available from three studies $[30,53,54]$. The PP of severe thrombocytopenia (61 cases) in patients with severe P. knowlesi malaria (77 cases) was $80 \%$ (95\% CI: 71-89\%, $\mathrm{I}^{2}: 0 \%$ ). The prevalence reported in the three individual studies of severe $P$. knowlesi malaria was consistent, ranging from $71 \%$ to $84 \%$. Overall, the PP of severe thrombocytopenia (440 cases) in patients with severe $P$. vivax and severe P. knowlesi malaria (885 cases) was 47\% (95\% CI: 33-61\%, I²: 96.5\%) (Figure 2).

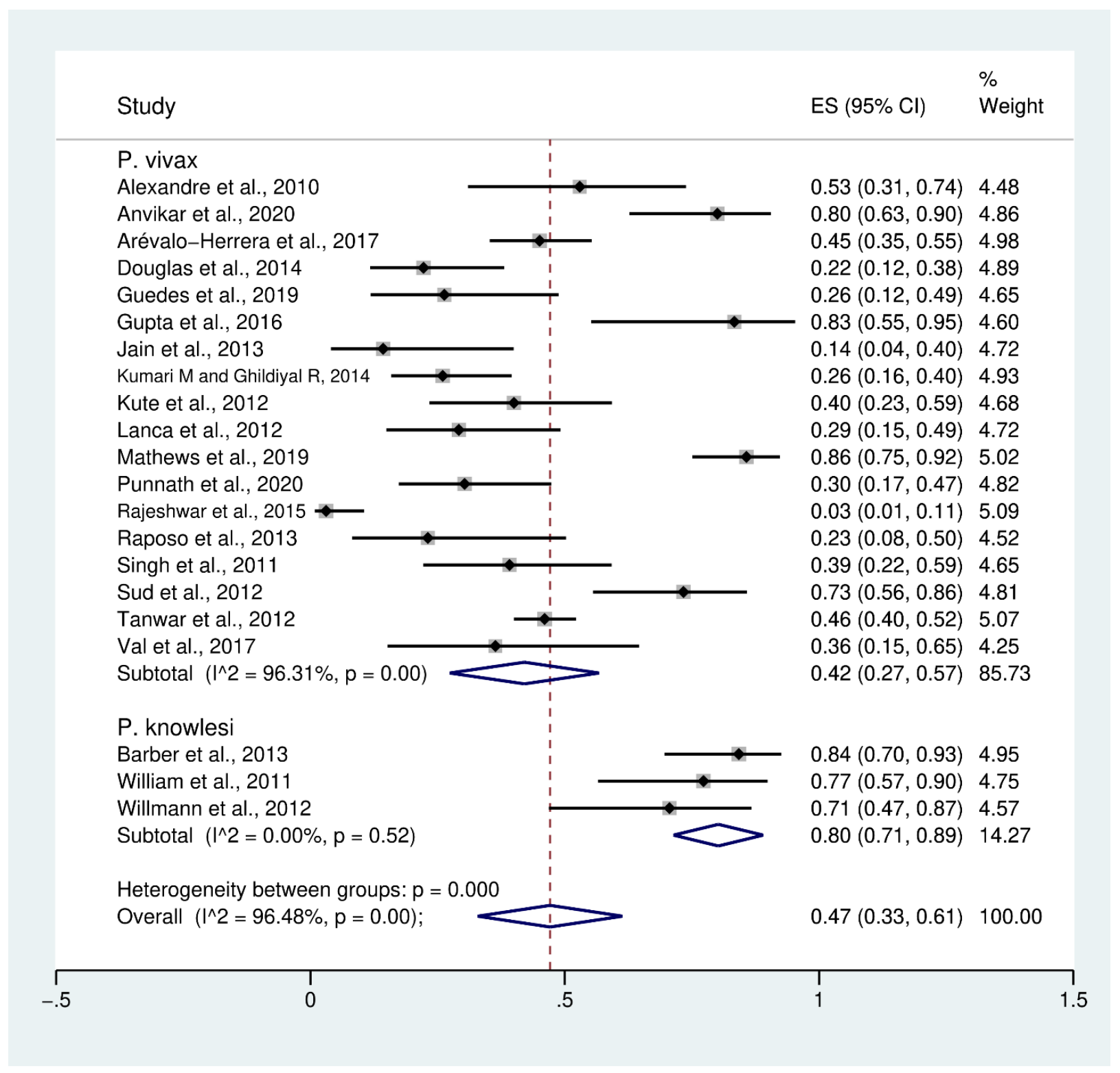

Figure 2. Prevalence of severe thrombocytopenia in patients with severe non- $P$. falciparum malaria. Abbreviations: ES, effect size; CI, confidence interval; black diamond symbol, point estimate; dashed line: pooled prevalence of severe thrombocytopenia; $\mathrm{I}^{2}$, level of heterogeneity; $p=0.00$ or less than 0.05 , significant heterogeneity. 


\subsection{Prevalence of Profound Thrombocytopenia in Severe Non-P. falciparum Malaria}

Data on profound thrombocytopenia (189 cases) in patients with severe P. vivax malaria (954 cases) from 12 studies $[10,23,26,27,30,32,36,38,41,45,48,49]$ were extracted and pooled in the meta-analysis of proportion. The PP of profound thrombocytopenia in patients with severe $P$. vivax malaria was $21 \%$ (95\% CI: $\left.14-28 \%, \mathrm{I}^{2}: 86.8 \%\right)$. The prevalence reported in individual studies varied highly (range: 2-67\%). Data on profound thrombocytopenia (13 cases) in patients with severe $P$. knowlesi malaria (60 cases) were available from two studies $[30,53]$. The PP of profound thrombocytopenia in patients with severe P. knowlesi malaria (77 cases) was 17\% (95\% CI: 8-26\%, I': 98.8\%). The prevalence reported in individual studies varied highly (range: 6-26\%). Overall, the PP of profound thrombocytopenia (202 cases) in patients with severe $P$. vivax and severe $P$. knowlesi malaria (1014 cases) was 20\% (95\% CI: $14-27 \%, \mathrm{I}^{2}: 85.2 \%$ ) (Figure 3 ).

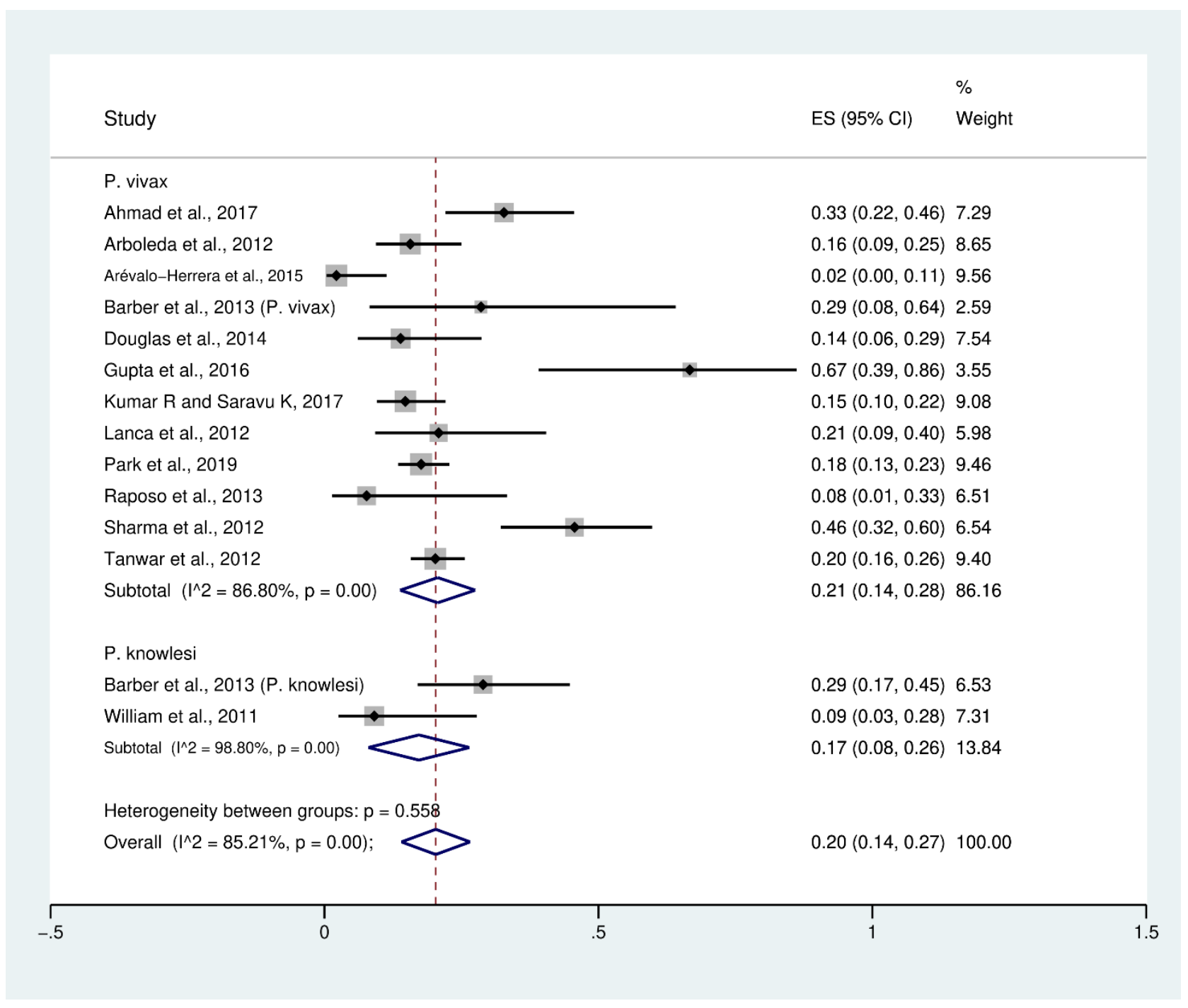

Figure 3. Prevalence of profound thrombocytopenia in patients with severe non- $P$. falciparum malaria. Abbreviations: ES, effect size; CI, confidence interval; black diamond symbol: point estimate; dashed line: pooled prevalence of severe thrombocytopenia; $\mathrm{I}^{2}$, level of heterogeneity; $p=0.00$ or less than 0.05 , significant heterogeneity.

\subsection{Difference in Platelet Counts between Patients with Severe and Uncomplicated Non-P. falciparum Malaria}

Data on mean platelet counts in patients with severe P. vivax malaria (557 cases) and uncomplicated $P$. vivax malaria (2451 cases) were available from eight studies $[27,30,33-35,38,43,45]$. 
The pooled weighted mean difference (WMD) showed that the platelet counts of patients with severe $P$. vivax malaria were significantly lower than those of patients with uncomplicated P. vivax malaria (WMD: $-26,500,95 \%$ CI: $-45,640$ to -7370 , Cochran's $\mathrm{Q}<0.0001$, $\mathrm{I}^{2}$ : $\left.98.1 \%\right)$. Data on mean platelet counts in patients with severe P. knowlesi malaria (124 cases) and uncomplicated P. knowlesi malaria (318 cases) were available from four studies $[29,30,53,54]$. The pooled WMD showed that the platelet counts of patients with severe $P$. knowlesi malaria were significantly lower than those of patients with uncomplicated P. knowlesi malaria (WMD: $-25,660,95 \%$ CI: $-34,150$ to $-17,180$, Cochran's $\mathrm{Q}<0.0001$, $\left.I^{2}: 89.9 \%\right)$. Overall, the pooled WMD showed the platelet counts of patients with severe non-P. falciparum malaria (681 cases) were significantly lower than those of patients with uncomplicated non- $P$. falciparum malaria (2769 cases) ( $p<0.001$, WMD: $-28,510,95 \%$ CI: $-40,350$ to $-16,680$, Cochran's $Q<0.0001, I^{2}: 97.7 \%$ ) (Figure 4).

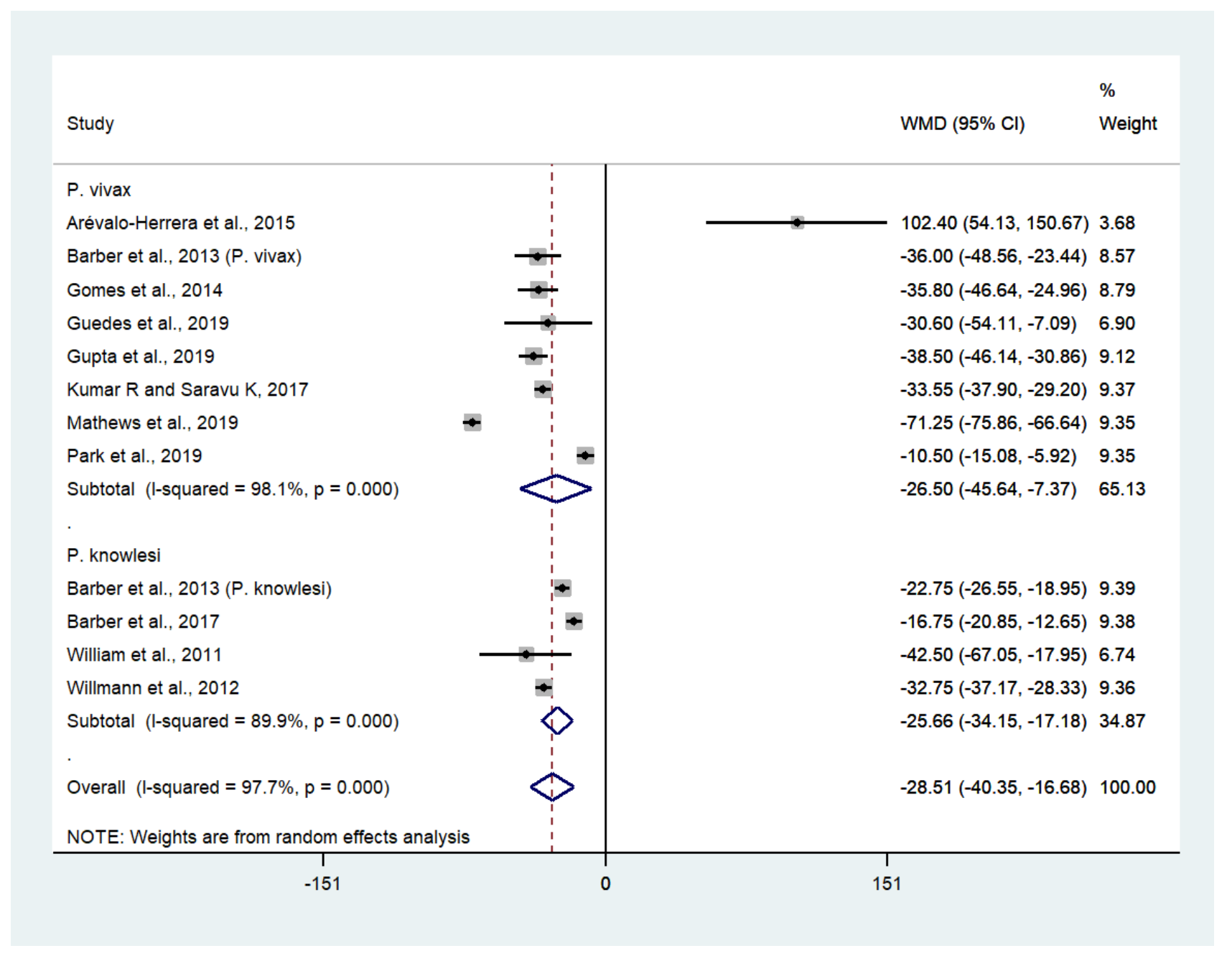

Figure 4. The difference in platelet counts between patients with severe and uncomplicated non- $P$. falciparum malaria. Abbreviations: WMD, weight mean difference; $\mathrm{CI}$, confidence interval; black diamond symbol, point estimate; solid line in the middle of the graph at 0 , zero effect size; dashed line: pooled WMD between the two groups; $\mathrm{I}^{2}$, level of heterogeneity; $p=0.00$ or less than 0.05 , significant heterogeneity.

3.6. Differences in Platelet Counts between Patients with Severe Non-P. falciparum and Severe P. falciparum Malaria

Data on mean platelet counts in patients with severe $P$. vivax malaria ( 252 cases) and severe P. falciparum malaria (576 cases) were available from seven studies [25,27,28,30,31,35,42]. The pooled WMD showed that the platelet counts in patients with severe $P$. vivax malaria and 
severe P. falciparum malaria were comparable (WMD: 16,300, 95\% CI: $-17,980$ to 21,230 , Cochran's $\left.\mathrm{Q}<0.001, \mathrm{I}^{2}: 88.3 \%\right)$. Data on mean platelet counts in patients with severe $P$. knowlesi malaria (85 cases) and severe P. falciparum malaria (26 cases) were available from two studies $[29,30]$. The pooled WMD showed that the platelet counts of patients with severe P. knowlesi and severe P. falciparum malaria were comparable (WMD: 3380, 95\% CI: -7990 to 1240 , Cochran's Q: 0.373, I²: 0\%). Overall, the pooled WMD showed that the platelet counts of patients with severe non- $P$. falciparum (337 cases) and severe $P$. falciparum malaria (610 cases) were comparable (WMD: $-3830,95 \%$ CI: -1390 to 6250, Cochran's $\mathrm{Q}<0.0001, \mathrm{I}^{2}: 85.2 \%$ ) (Figure 5).

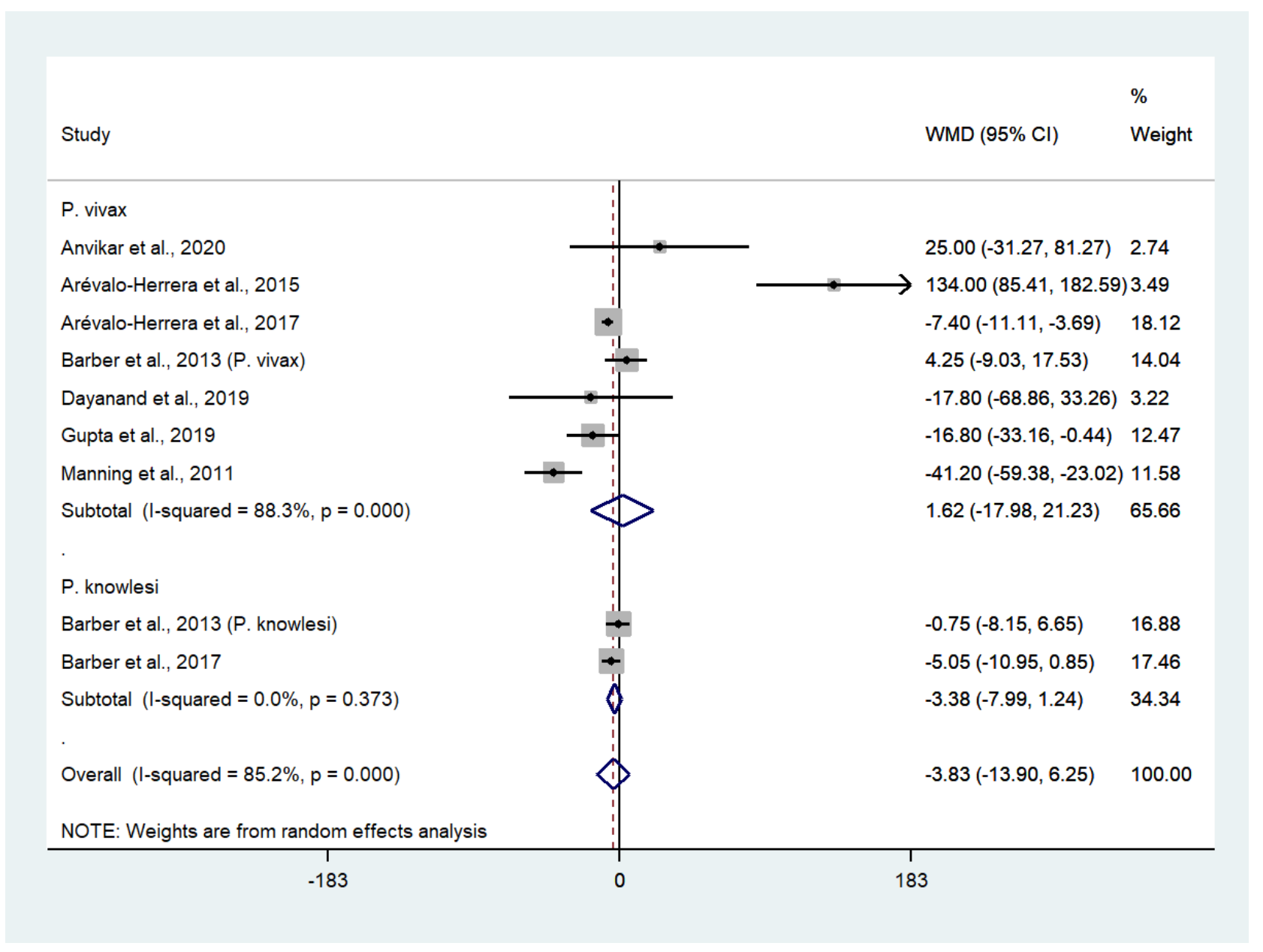

Figure 5. The absence of difference in platelet counts between patients with severe non- $P$. falciparum and severe $P$. falciparum malaria. Abbreviations: WMD, weight mean difference; CI, confidence interval; black diamond symbol, point estimate; solid line in the middle of the graph at 0 , zero effect size; dashed line: pooled WMD between the two groups; $\mathrm{I}^{2}$, level of heterogeneity; $p=0.00$ or less than 0.05 , significant heterogeneity.

\subsection{Meta-Regression Analysis}

A meta-regression analysis of the difference in platelet counts between patients with severe and uncomplicated non-P. falciparum malaria was performed using age, male ratio, and parasite counts as co-variates. The results of the univariate meta-regression analysis showed that the difference in platelet counts between the two groups was not confounded by age, male ratio, or parasite counts ( $p>0.05$, Figures S1-S3). A meta-regression analysis of the difference in platelet counts between patients with severe non-P. falciparum and severe $P$. falciparum malaria was also performed using age, male ratio, and parasite counts as co-variates. The results of the univariate meta-regression analysis showed that the 
difference in platelet counts between the two groups was not confounded by age, male ratio, or parasite counts $(p>0.05$, Figures S4-S6).

3.8. Risk of Severe or Profound Thrombocytopenia in Patients with Severe Non-P. falciparum Malaria Compared with Uncomplicated Non-P. falciparum Malaria

Data on severe or profound thrombocytopenia in patients with severe $P$. vivax malaria (403 cases) and uncomplicated $P$. vivax malaria (1647 cases) were available from five studies $[30,34,38,45,54]$. Patients with severe $P$. vivax malaria had significantly higher odds of severe or profound thrombocytopenia than those with uncomplicated $P$. vivax malaria (OR: 3.21, 95\% CI: 2.19-4.69, Cochran's Q: 0.34, $\mathrm{I}^{2}:$ 10.2\%). Data on severe thrombocytopenia in patients with severe $P$. knowlesi malaria (55 cases) and uncomplicated $P$. knowlesi malaria (185 cases) were available from two studies [30,54]. Patients with severe $P$. knowlesi malaria had significantly higher odds of severe or profound thrombocytopenia than those with uncomplicated P. knowlesi malaria (OR: 1.92, 95\% CI: 1.18-3.11, Cochran's Q: 0.445, I²: 0\%). Overall, the odds of severe or profound thrombocytopenia were significantly higher in patients with severe non-P. falciparum malaria compared with those with uncomplicated non-P. falciparum malaria (OR: 2.64, 95\% CI: 1.85-3.77, Cochran's Q: 0.219, I²: 28.7\%) (Figure 6).

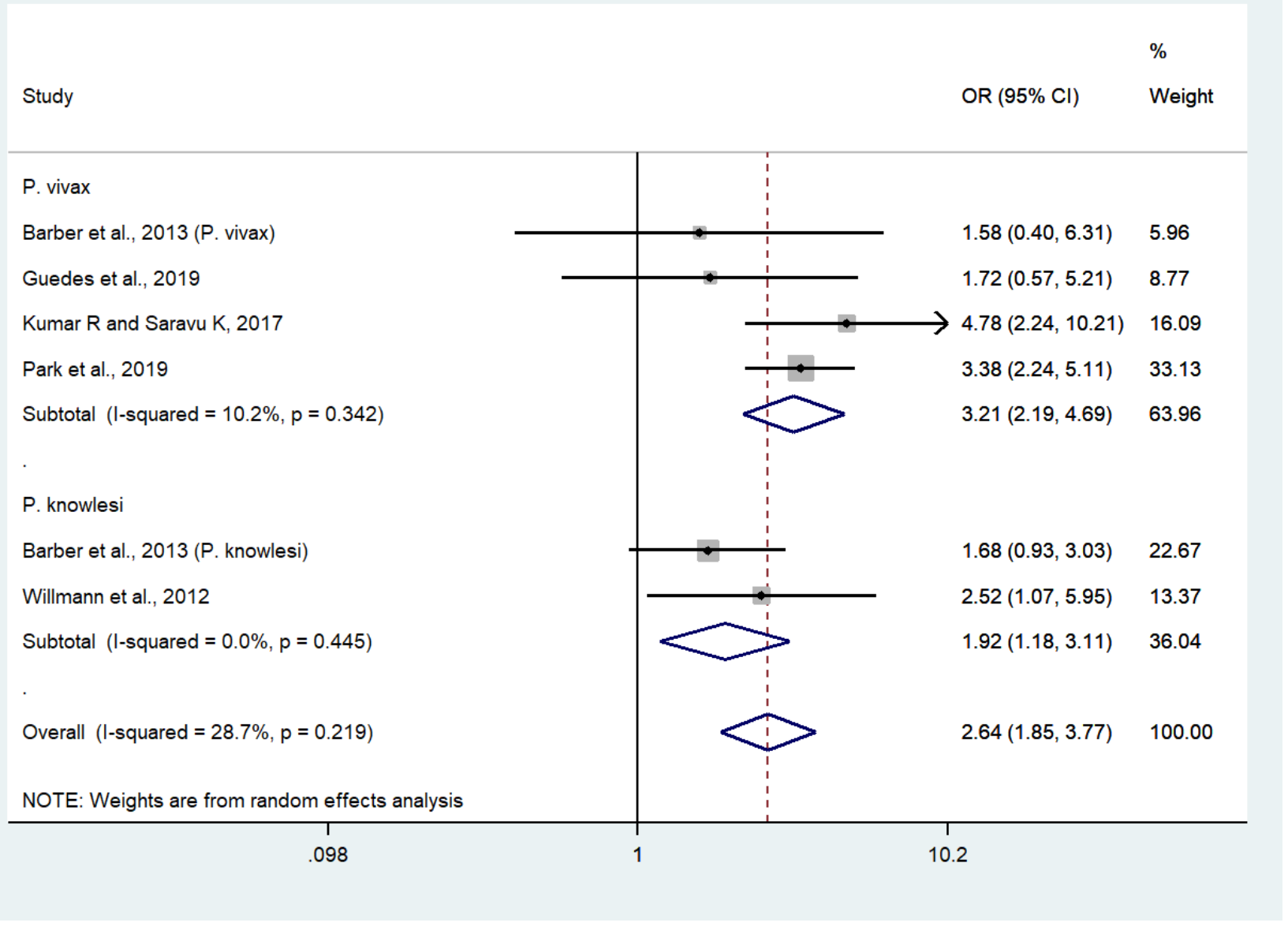

Figure 6. Risk of severe or profound thrombocytopenia in patients with severe non- $P$. falciparum malaria compared with uncomplicated non-P. falciparum malaria. Abbreviations: OR, odds ratio; CI, confidence interval; black diamond symbol, point estimate; solid line in the middle of the graph at 1, non-difference of effect size; dashed line: pooled OR between the two groups; $\mathrm{I}^{2}$, level of heterogeneity; $p=0.00$ or less than 0.05 , significant heterogeneity. 
3.9. Risk of Severe or Profound Thrombocytopenia in Patients with Severe Non-P. falciparum Malaria Compared with Severe P. falciparum Malaria

Data on severe or profound thrombocytopenia in patients with severe $P$. vivax malaria (381 cases) and severe $P$. falciparum malaria (396 cases) were available from seven studies $[24,25,27,28,30,38,46]$. The odds of severe or profound thrombocytopenia in patients with severe $P$. vivax and severe $P$. falciparum malaria were comparable (OR: 1.01, 95\% CI: 0.73-1.40, Cochran's Q: 0.839, I ${ }^{2}: 0 \%$ ). Data on severe thrombocytopenia in patients with severe $P$. knowlesi malaria (32 cases) and severe P. falciparum malaria (10 cases) were available from one study [30]. The results showed that the odds of severe or profound thrombocytopenia were comparable in patients with severe $P$. knowlesi and severe P. falciparum malaria (OR: 1.09, 95\% CI: 0.42-2.83). Overall, no significant difference was observed in the odds of severe or profound thrombocytopenia between patients with severe non-P. falciparum and severe P. falciparum malaria (OR: 1.02, 95\% CI: 75-1.39, Cochran's Q: 0.905, I²: 0\%) (Figure 7).

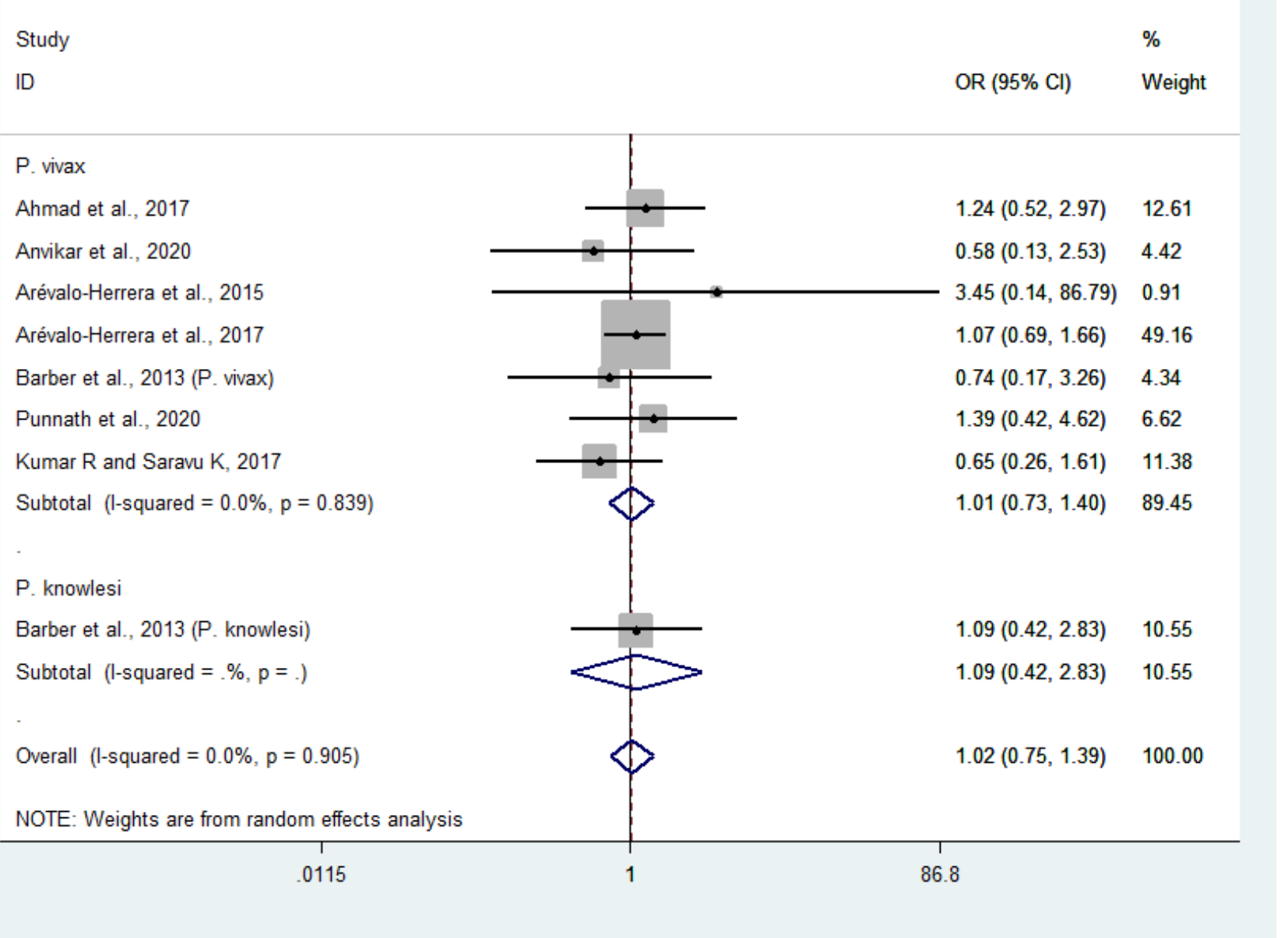

Figure 7. Risk of severe or profound thrombocytopenia in patients with severe non- $P$. falciparum malaria compared with severe P. falciparum malaria. Abbreviations: OR, odds ratio; CI, confidence interval; black diamond symbol, point estimate; solid line in the middle of the graph at 1 , non-difference of effect size; dashed line: pooled OR between the two groups; $\mathrm{I}^{2}$, level of heterogeneity; $p=0.00$ or less than 0.05 , significant heterogeneity.

\subsection{Deaths Related to Severe or Profound Thrombocytopenia}

Data on deaths from severe P. vivax malaria were available for extraction. Deaths resulting from severe $P$. vivax malaria in patients with severe or profound thrombocytopenia were investigated. Mortality related to severe thrombocytopenia in patients with severe 
$P$. vivax malaria (63 cases with deaths, in three studies $[28,39,50])$ was $4 \%(95 \% \mathrm{CI}:-2 \%$ to $10 \%)$, while mortality related to profound thrombocytopenia in patients with severe P. vivax malaria (21 cases with deaths) was $24 \%$ (95\% CI: 0-45\%) [49].

\subsection{Publication Bias}

Publication bias was assessed by inspecting funnel plot asymmetry. Asymmetry was confirmed using an Egger's test. The funnel plot of studies reporting the outcomes of patients with severe thrombocytopenia was asymmetrical, reflecting small-study effects (Figure 8 ). This asymmetry was confirmed by the Egger's test, demonstrating the existence of small-study effects across the 21 included studies ( $p=0.041$, $t$-statistic: 2020, coefficient: 4.48, standard error: 2.04). A contour-enhanced funnel plot was generated and showed that the asymmetry of the funnel plot resulted from publication bias in most studies (Figure 9).

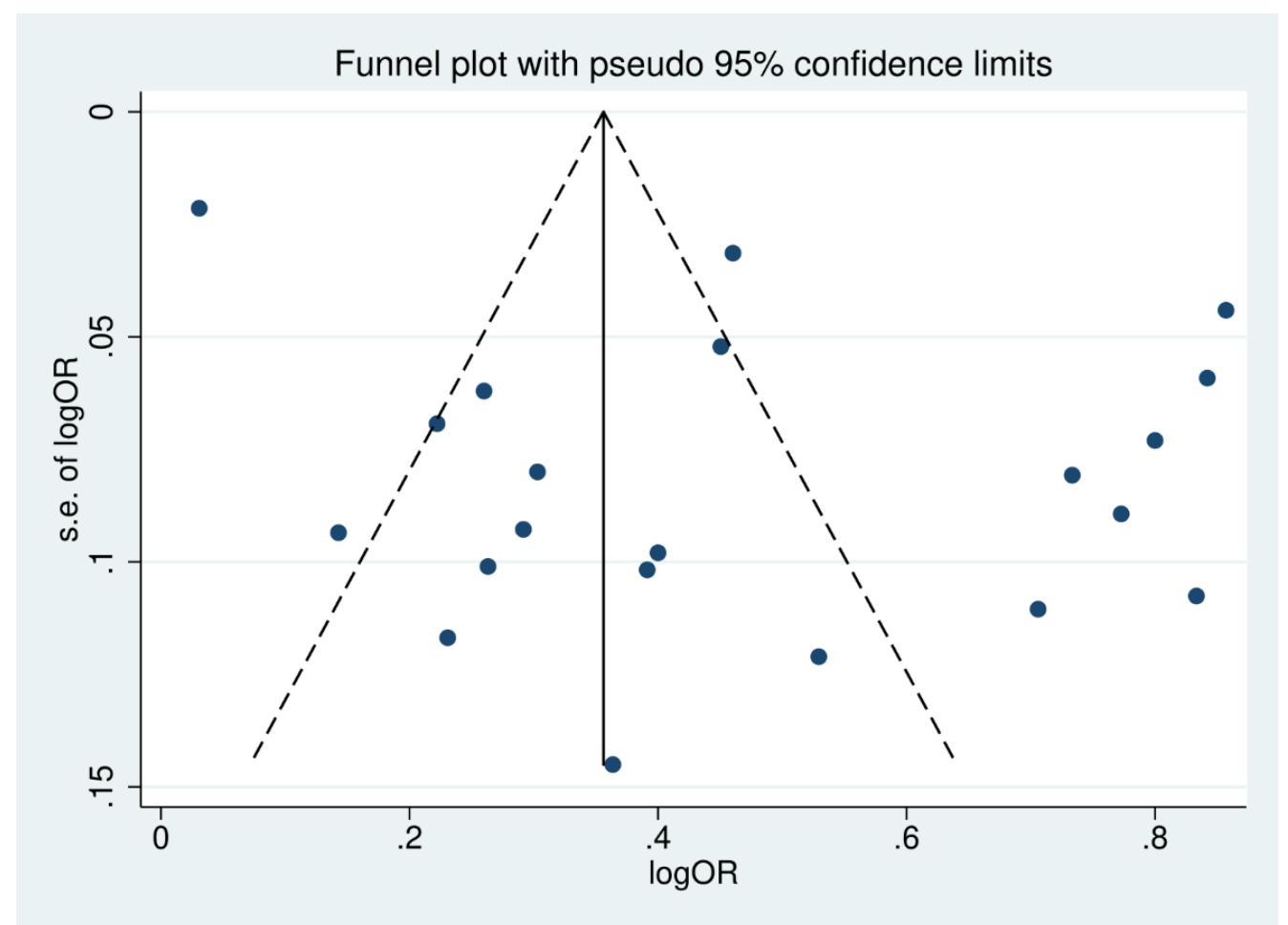

Figure 8. Funnel plot of included studies. The funnel plots showed the asymmetrical distribution of the $\log \mathrm{OR}$ and the standard error (se) of logOR among studies reporting outcomes of patients with severe thrombocytopenia. 


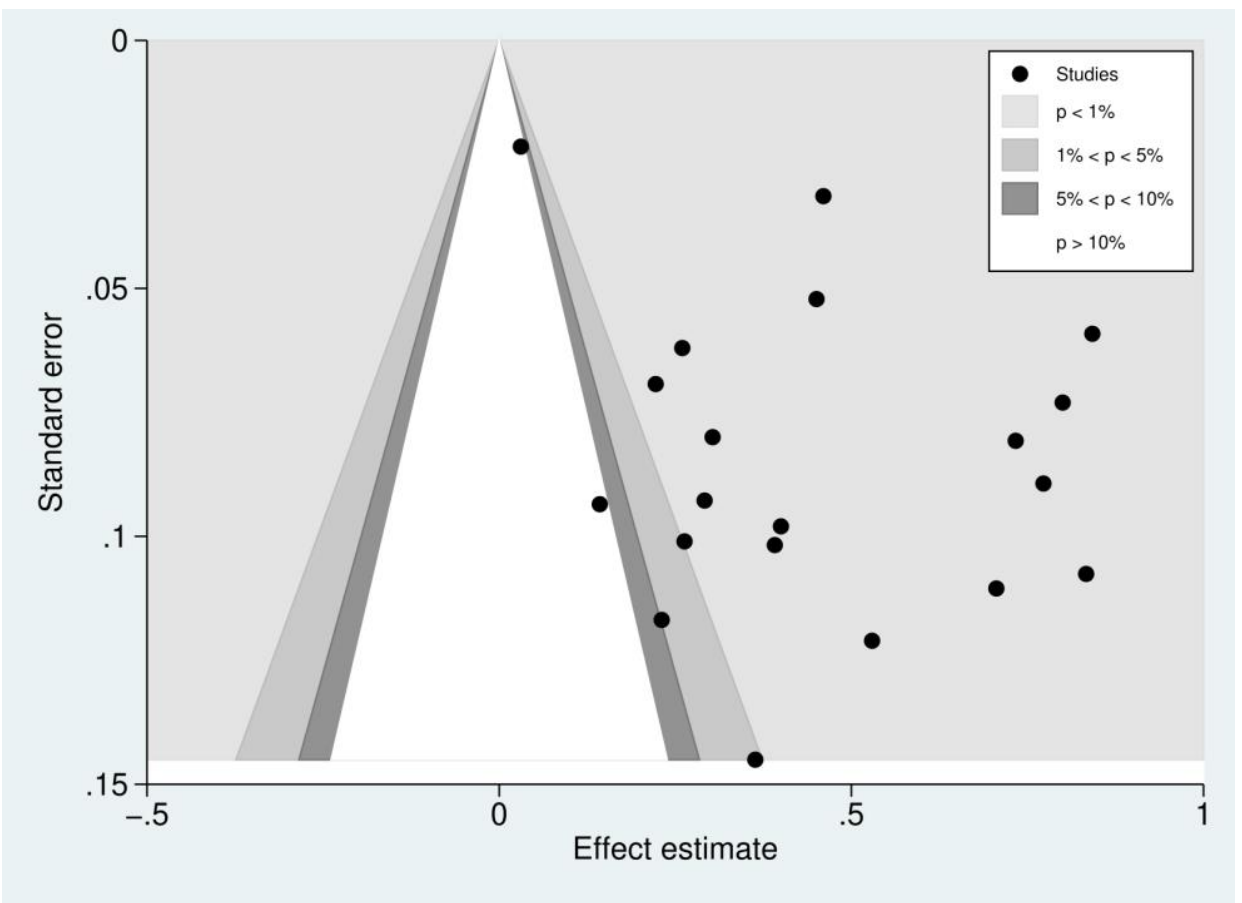

Figure 9. Contour-enhanced funnel plot of included studies. Funnel plot of included studies. The plots showed the distribution of the effect estimate $(\log \mathrm{OR})$ and the standard error (se) of $\log \mathrm{OR}$ among studies reporting outcomes of patients with severe thrombocytopenia. Most of the effect estimates (black dots) located in the significant area $(p<0.01,1 \%)$ indicated that the asymmetry of the funnel plot resulted from publication bias.

\section{Discussion}

Thrombocytopenia frequently occurs in individuals with $P$. falciparum and P. vivax malaria $[55,56]$. The greatest risks of severe thrombocytopenia were observed in individuals with $P$. falciparum single or mixed infections, accounting for $>40 \%$ of cases [57]. In addition, the risk of severe thrombocytopenia was higher in patients with $P$. falciparum malaria than those with $P$. vivax malaria [57]. Moreover, severe malaria and mortality were more frequent in patients with $P$. falciparum malaria and severe thrombocytopenia [57-59]. To the best of our knowledge, this is the first systematic review and meta-analysis to examine severe and profound thrombocytopenia among individuals with severe non- $P$. falciparum malaria. Our results illustrate the high prevalence of severe thrombocytopenia in patients with severe $P$. vivax malaria $(42 \%)$ and severe $P$. knowlesi malaria $(80 \%)$. In addition to severe thrombocytopenia, we found that the PP of profound thrombocytopenia was low in patients with severe $P$. vivax malaria $(21 \%)$ and severe $P$. knowlesi malaria $(17 \%)$. The $\mathrm{PP}$ of severe and profound thrombocytopenia in patients with severe $P$. vivax malaria could not be precisely estimated because of the high heterogeneity among the included studies $\left(\mathrm{I}^{2}>86 \%\right)$. In addition, the PP of severe thrombocytopenia in patients with severe P. knowlesi malaria could be reliably estimated because of the homogeneity of the included studies $\left(\mathrm{I}^{2}: 0 \%\right)$. Nevertheless, we caution that the homogeneity among studies reporting the outcomes of patients with severe thrombocytopenia in $P$. knowlesi malaria could result from the relatively small number of included studies. Severe thrombocytopenia is not currently considered an indicator of severe malaria according to the WHO criteria [60]. Interestingly, in light of our analysis of severe and profound thrombocytopenia in patients with severe non- $P$. falciparum malaria, it may be worth investigating individuals with severe malaria and severe or profound thrombocytopenia. Clinicians managing patients with malaria should be aware of the risks, as these individuals are more likely to die than patients with higher platelet counts [14]. Furthermore, a previous study suggested that 
profound thrombocytopenia should be used as a severity criterion because it is a risk factor for mortality [57].

Thrombocytopenia is a common feature of malaria resulting from P. falciparum, P. vivax, and P. knowlesi infection $[29,30,55]$. The degree of thrombocytopenia relates to the high parasitemia of Plasmodium species [61]. Although thrombocytopenia is common in Plasmodium infections, significant bleeding is often unrelated to the level of thrombocytopenia [27]. Therefore, arguments have been made for routine platelet transfusions in malaria patients [62] and not defining thrombocytopenia as a clinical manifestation of severe malaria [60]. The potential diagnostic utility of severe or profound thrombocytopenia in individual patients is for the rapid treatment of severe malaria, as these patients may require additional support for clinical management [63]. The present study showed that the mean platelet counts of patients with severe $P$. vivax and severe $P$. knowlesi malaria were lower than those of patients with uncomplicated $P$. vivax and $P$. knowlesi malaria. Although high heterogeneity was present in the pooled analysis, these results demonstrated the significant diagnostic value of lower platelet counts in patients with severe non- $P$. falciparum malaria compared with patients with uncomplicated malaria. In the two-by-two meta-analysis of proportion, individuals with severe $P$. vivax malaria had more than three times the odds of developing severe or profound thrombocytopenia than those with uncomplicated P. vivax malaria. Lower odds of severe or profound thrombocytopenia were observed in patients with severe $P$. knowlesi malaria. The pooled ORs from the meta-analysis were reliable because of the low heterogeneity among the included studies. From the results of meta-analysis, most of the included studies showed lower mean platelet counts in patients with severe $P$. vivax malaria than in those with uncomplicated malaria $[30,33-35,38,43,45]$, while only Arévalo-Herrera et al., 2015 [27] demonstrated higher mean platelet counts in patients with severe $P$. vivax malaria. Arévalo-Herrera et al., 2015 reported a wider range of platelet counts in patients with severe $P$. vivax malaria $(82,000-660,000$ cells $/ \mu \mathrm{L})$ than in those with uncomplicated $P$. vivax malaria, but no difference in the risk of spontaneous bleeding associated with thrombocytopenia [27]. Trends in mean platelet counts in patients with severe $P$. knowlesi malaria were similar to those in patients with severe $P$. vivax malaria: all included studies reported a significantly lower mean platelet count in these patients than in those with uncomplicated P. knowlesi malaria. Thrombocytopenia in patients with $P$. knowlesi infections is common, and platelet counts were lowest in cases of severe disease [53]. However, patients with severe P. knowlesi malaria exhibited higher mean platelet counts than those with severe $P$. vivax malaria.

We found no significant differences in the mean platelet counts of patients with severe P. vivax/P. knowlesi malaria and severe P. falciparum malaria. The two-by-two meta-analysis of proportion confirmed that neither severe nor profound thrombocytopenia was significantly different in patients with severe $P$. vivax/P. knowlesi malaria and severe $P$. falciparum malaria. The meta-analysis results were reliable because of the low heterogeneity in the pooled analysis of proportion. Our results demonstrated that severe and profound thrombocytopenia might affect the risk of death in individuals with severe $P$. vivax malaria. The data reported by four included studies $[28,39,49,50]$ demonstrated that 84 individuals with severe $P$. vivax malaria exhibited severe and profound thrombocytopenia. Infection by other Plasmodium species, including $P$. malariae and $P$. ovale malaria, resulted in severe thrombocytopenia less frequently than $P$. vivax malaria [64,65] and P. falciparum malaria [65]. A previous study found that severe thrombocytopenia was significantly more common in patients with P. ovale wallikeri infection compared with those with P. ovale curtisi infection [66]. In $P$. falciparum infection, patients with profound thrombocytopenia were five times more likely to die than patients with higher platelet counts, suggesting that thrombocytopenia might be a marker of disease severity in patients with falciparum malaria [14,57]. The previous study that enrolled patients with $P$. falciparum and $P$. vivax suggested that severe thrombocytopenia had a sensitivity and specificity of $65.6 \%$ and $70.6 \%$, respectively, to discriminate against severe malaria. Therefore, it is valuable to further investigate the sensitivity and specificity of thrombocytopenia using large longitudinal studies to conclude the 
performance of using platelet as an indicator for severe non-P. falciparum malaria. In light of our analyses for non-P. falciparum malaria, the mortality rate in patients with profound thrombocytopenia was higher than those with severe thrombocytopenia, suggesting the possibility of using thrombocytopenia to identify the risk of death in patients with severe non-P. falciparum malaria.

The pathogenic mechanisms underlying thrombocytopenia and the mechanisms through which platelet numbers affect severe malaria and death remain unclear. However, previous studies have shown that endothelial activation [67] results in platelet aggregation and clearance from circulation by the von Willebrand Factor (vWF) [68]. The role of platelet aggregation was supported by the finding that individuals with severe $P$. falciparum malaria deficient in a disintegrin and metalloprotease with thrombospondin type I repeats, member 13 (ADAMTS13), had higher frequencies of severe thrombocytopenia and other complications [69]. The high platelet consumption and activation observed in severe and fatal malaria cases may contribute to severe thrombocytopenia as platelet-expressed Toll-like receptors bind $P$. falciparum molecules and release prepackaged inflammatory mediators, leading to decreased signaling and inflammatory responses [70,71]. Another potential mechanism of severe thrombocytopenia-related malaria severity involves nitric oxide (NO). $\mathrm{NO}$ is a mediator of platelet homeostasis, and previous studies found a reduction in NO bioavailability in individuals with severe and fatal malaria [72].

In addition to identifying new candidate makers for severe malaria, new diagnostic tools for detecting malaria parasites are challenging. Recently, magnetic resonance relaxometry (MRR) [73-75], on-chip nuclear magnetic resonance (NMR) [76], rotating-crystal magneto-optical detection (RMOD) [77], and fluorescent blue-ray optical devices [78] have emerged as high-sensitivity malaria diagnostic tools to detect low parasite density in asymptomatic individuals. These new diagnostic tools will help increase the detection of malaria parasites and provide early treatment to patients, which can reduce malaria transmission, reducing the rate of misdiagnosis or mis-treatment that can facilitate severe malaria or anti-malarial drug resistance.

Biological and observational studies of non- $P$. falciparum malaria, particularly of P. knowlesi are fragmented, and is a research gap for malaria studies. The present study, Section 3.6 could only be derived from seven P. vivax studies and two P. knowlesi studies. Section 3.9 could only be derived from seven $P$. vivax studies and one P. knowlesi study, while Section 3.10 could only be derived from three $P$. vivax studies. It would be interesting to determine whether such comparability in the severity or profoundness of thrombocytopenia and related outcomes was due to the limitations in the available studies or unique biological traits that exist within non-P. falciparum species that induce host responses. The detection of human cases of $P$. knowlesi demonstrated an ongoing increase despite effective control programs to disrupt the transmission of human-only Plasmodium species [77]. Some notable concern in terms of a potential increase in human infections with $P$. knowlesi malaria can be found in the changing patterns in land use where spillover opportunities for human infection due to increased contact with natural reservoirs and infected vectors have been aggravated. In addition, the range and distribution of primary hosts and vectors including their bionomics are still lacking in data [78]. These are strong rationales to conduct further studies on the parasite, vector, and host biology in non-P. falciparum malaria and host immunological responses to the same.

Our study had several limitations. First, the data on platelet counts and the frequency of severe or profound thrombocytopenia could only be extracted from studies of patients with severe $P$. vivax and severe $P$. knowlesi malaria. Therefore, the prevalence of severe or profound thrombocytopenia was not assessed among patients with severe P. malariae, P. ovale, or mixed malaria. Second, the prevalence of severe or profound thrombocytopeniarelated death could not be precisely estimated because of the limited number of included studies. Despite these limitations, the presence of severe or profound thrombocytopenia in malaria patients may support the diagnosis of malaria, particularly in patients with severe non-P. falciparum malaria, because low levels of parasitemia are frequently encountered in 
routine diagnosis. In addition, severe or profound thrombocytopenia could be used as an indicator of increased risk of poor outcome and mortality if there are delays in diagnosis. Our results suggest that severe and/or profound thrombocytopenia should be included in the WHO criteria for severe non-P. falciparum malaria because a high prevalence of severe thrombocytopenia was observed in these patients ( $47 \%$ for severe thrombocytopenia and $20 \%$ for profound thrombocytopenia).

The present systematic review provided the new insight into the clinical relevance of severe and profound thrombocytopenia in diagnosing and managing non-P. falciparum malaria. Severe thrombocytopenia should serve as a warning sign of increased chances of complications. Meanwhile, profound thrombocytopenia should serve as a marker of mortality among patients with non-P. falciparum malaria.

\section{Conclusions}

A meta-analysis provides enhanced statistical power, thereby providing more robust and reliable knowledge of the effects of severe non-P. falciparum malaria and thrombocytopenia. Our results suggest that individuals with severe non- $P$. falciparum malaria, particularly $P$. vivax and $P$. knowlesi malaria, with severe or profound thrombocytopenia should be regarded as high-risk patients and should subsequently be treated for severe malaria according to the current WHO guidelines. In addition, severe or profound thrombocytopenia coupled with other clinical and microscopy parameters, may significantly improve malaria diagnosis, provide timely treatment for malaria infections, and reduce the morbidity and mortality of severe non- $P$. falciparum malaria. Observational studies and research on the parasite, vector, and host biology and outcomes for non-P. falciparum malaria is still fragmented. Moreover, the changing patterns of land use that increase the exposure of natural reservoirs and infected vectors, range distribution of hosts, and vectors, including the biomics of non- $P$. falciparum malaria, require further and more in-depth exploration.

Supplementary Materials: The following are available online at https:/ /www.mdpi.com/article/10 $.3390 /$ biology10121275/s1, Figure S1. Results of univariate meta-regression analysis showing differences in platelet counts between patients with severe and uncomplicated non-P. falciparum malaria using age as a covariate; Figure S2. Results of univariate meta-regression analysis showing differences in platelet counts between patients with severe and uncomplicated non-P. falciparum malaria using the male ratio as a covariate; Figure S3. Results of univariate meta-regression analysis showing differences in platelet counts between patients with severe and uncomplicated non-P. falciparum malaria using parasite count ratio as a covariate; Figure S4. Results of univariate meta-regression analysis showing differences in platelet counts between patients with severe non-P. falciparum and severe P. falciparum malaria using age as a covariate; Figure S5. Results of univariate meta-regression analysis showing differences in platelet counts between patients with severe non-P. falciparum and severe P. falciparum malaria using the male ratio as a covariate; Figure S6. Results of univariate meta-regression analysis showing differences in platelet counts between patients with severe non-P. falciparum and severe P. falciparum malaria using parasite count ratio as a covariate; Table S1. Characteristics of the included studies; Table S2. Risk of bias in the included studies. PRISMA Checklist S1. Guidelines for reporting systematic review and meta-analysis.

Author Contributions: Conceptualization, A.M. and M.K.; methodology, A.M. and M.K.; software, M.K.; validation, F.R.M., G.D.J.M. and K.U.K.; formal analysis, M.K.; investigation, M.K.; resources, P.W.; data curation, W.M.; writing — original draft preparation, M.K.; writing—review and editing, A.M.; visualization, M.K.; supervision, P.W.; project administration, M.K.; funding acquisition, M.K. All authors have read and agreed to the published version of the manuscript.

Funding: This research was partially supported by the New Strategic Research (P2P) Project, Walailak University, Thailand. The funders played no role in the collection, analysis, and interpretation of the data.

Institutional Review Board Statement: Not applicable.

Informed Consent Statement: Not applicable. 
Data Availability Statement: All data relating to the present study are available in this manuscript.

Acknowledgments: This research was financially supported by the new strategic research project (P2P) fiscal year 2022, Walailak University, Thailand.

Conflicts of Interest: The authors declare that there are no conflict of interest regarding the publication of this article.

\section{References}

1. van der Meijden, P.E.J.; Heemskerk, J.W.M. Platelet biology and functions: New concepts and clinical perspectives. Nat. Rev. Cardiol. 2019, 16, 166-179. [CrossRef]

2. Das, S.; Rajkumari, N.; Chinnakali, P. A comparative study assessing the effect of haematological and biochemical parameters on the pathogenesis of malaria. J. Parasit. Dis. 2019, 43, 633-637. [CrossRef]

3. Sari, N.D.; Yoruk, G. Retrospective evaluation of 31 malaria cases hospitalized in our clinic between 2012-2018. Turk. Parazitol. Derg. 2019, 43, 170-174. [CrossRef] [PubMed]

4. Nwaneri, D.; Oladipo, O.; Ifebi, E.; Oviawe, O.; Asemota, O.; Ogboghodo, B.; Israel-Aina, Y.; Sadoh, A. Haematological parameters and spleen rate of asymptomatic and malaria negative children in Edo south district, Nigeria. Ann. Glob. Health 2020, 86, 62. [CrossRef]

5. Kochar, D.K.; Das, A.; Kochar, A.; Middha, S.; Acharya, J.; Tanwar, G.S.; Gupta, A.; Pakalapati, D.; Garg, S.; Saxena, V.; et al. Thrombocytopenia in Plasmodium falciparum, Plasmodium vivax and mixed infection malaria: A study from Bikaner (Northwestern India). Platelets 2010, 21, 623-6277. [CrossRef]

6. $\quad$ Leal-Santos, F.A.; Silva, S.B.; Crepaldi, N.P.; Nery, A.F.; Martin, T.O.; Alves-Junior, E.R.; Fontes, C.J. Altered platelet indices as potential markers of severe and complicated malaria caused by Plasmodium vivax: A cross-sectional descriptive study. Malar. J. 2013, 12, 462. [CrossRef]

7. Supanaranond, W.; Davis, T.M.; Dawes, J.; Silamut, K.; Vilaiwanna, N.; White, N.J. In-vivo platelet activation and anomalous thrombospondin levels in severe falciparum malaria. Platelets 1992, 3, 195-200. [CrossRef]

8. Kochar, D.K.; Das, A.; Kochar, A.; Middha, S.; Acharya, J.; Tanwar, G.S.; Gupta, A.; Pakalapati, D.; Garg, S.; Saxena, V.; et al. Platelet accumulation in brain microvessels in fatal pediatric cerebral malaria. J. Infect. Dis. 2003, 187, 461-466.

9. Lacerda, M.V.; Mourao, M.P.; Coelho, H.C.; Santos, J.B. Thrombocytopenia in malaria: Who cares? Mem. Inst. Oswaldo Cruz. 2011, 106 (Suppl. 1), 52-63. [CrossRef]

10. Tanwar, G.S.; Khatri, P.C.; Chahar, C.K.; Sengar, G.S.; Kochar, A.; Tanwar, G.; Chahar, S.; Khatri, N.; Middha, S.; Acharya, J.; et al. Thrombocytopenia in childhood malaria with special reference to P. vivax monoinfection: A study from Bikaner (Northwestern India). Platelets 2012, 23, 211-216. [CrossRef] [PubMed]

11. Thapa, R.; Biswas, B.; Mallick, D.; Sardar, S.; Modak, S. Childhood Plasmodium vivax malaria with severe thrombocytopenia and bleeding manifestations. J. Pediatr. Hematol. Oncol. 2009, 31, 758-759. [CrossRef]

12. Punyagupta, S.; Srichaikul, T.; Nitiyanant, P.; Petchclai, B. Acute pulmonary insufficiency in falciparum malaria: Summary of 12 cases with evidence of disseminated intravascular coagulation. Am. J. Trop. Med. Hyg. 1974, 23, 551-559. [CrossRef]

13. WHO. Guidelines for the Treatment of Malaria 2015. Available online: https://www.who.int/docs/default-source/documents/ publications /gmp/guidelines-for-the-treatment-of-malaria-eng.pdf?sfvrsn=a0138b77_2 (accessed on 25 July 2020).

14. Hanson, J.; Phu, N.H.; Hasan, M.U.; Charunwatthana, P.; Plewes, K.; Maude, R.J.; Prapansilp, P.; Kingston, H.W.; Mishra, S.K.; Mohanty, S.; et al. The clinical implications of thrombocytopenia in adults with severe falciparum malaria: A retrospective analysis. BMC Med. 2015, 13, 97. [CrossRef]

15. Naing, C.; Whittaker, M.A. Severe thrombocytopaenia in patients with vivax malaria compared to falciparum malaria: A systematic review and meta-analysis. Infect. Dis. Poverty 2018, 7, 10. [CrossRef]

16. Wells, G.A.; Shea, B.; O'Connell, D.; Peterson, J.; Welch, V.; Losos, M.; Tugwell, P. The Newcastle-Ottawa Scale (NOS) for Assessing the Quality of Nonrandomised Studies in Meta-Analyses 2019. Available online: http://www.ohri.ca/programs/ clinical_epidemiology / oxford.asp (accessed on 25 July 2020).

17. DerSimonian, R.; Laird, N. Meta-analysis in clinical trials. Control. Clin. Trials 1986, 7, 177-188. [CrossRef]

18. Kotepui, M.; Kotepui, K.U.; De Jesus Milanez, G.; Masangkay, F.R. Plasmodium spp. mixed infection leading to severe malaria: A systematic review and meta-analysis. Sci. Rep. 2020, 10, 11068. [CrossRef]

19. Kotepui, M.; Kotepui, K.U.; Milanez, G.D.; Masangkay, F.R. Severity and mortality of severe Plasmodium ovale infection: A systematic review and meta-analysis. PLOS ONE 2020, 15, e0235014. [CrossRef]

20. Kotepui, M.; Kotepui, K.U.; Milanez, G.D.; Masangkay, F.R. Prevalence of severe Plasmodium knowlesi infection and risk factors related to severe complications compared with non-severe $P$. knowlesi and severe P. falciparum malaria: A systematic review and meta-analysis. Infect. Dis. Poverty 2020, 9, 106. [CrossRef]

21. Kotepui, M.; Kotepui, K.U.; Milanez, G.J.; Masangkay, F.R. Prevalence and risk factors related to poor outcome of patients with severe Plasmodium vivax infection: A systematic review, meta-analysis, and analysis of case reports. BMC Infect. Dis. 2020, 20, 363. [CrossRef]

22. Kotepui, M.K.K.; Milanez, G.D.; Masangkay, F.R. Global prevalence and mortality of severe Plasmodium malariae infection: A systematic review and meta-analysis. Malar. J. 2020, 10, 11068. [CrossRef] 
23. Ahmad, S.; Shirazi, N.; Bhat, N.K.; Dhar, M.; Mittal, G.; Mittal, M.; Kaeley, N.; Kumar, M. A hospital-based retrospective comparative study of complications, outcomes, clinical and laboratory parameters of malaria with and without neurological involvement. Mediterr. J. Hematol. Infect. Dis. 2017, 9, e2017006. [CrossRef]

24. Alexandre, M.A.; Ferreira, C.O.; Siqueira, A.M.; Magalhães, B.L.; Mourão, M.P.G.; Lacerda, M.V.; Alecrim, M.D.G.C. Severe Plasmodium vivax malaria, Brazilian Amazon. Emerg. Infect. Dis. 2010, 16, 1611-1614. [CrossRef]

25. Anvikar, A.R.; van Eijk, A.M.; Shah, A.; Upadhyay, K.J.; Sullivan, S.A.; Patel, A.J.; Joshi, J.M.; Tyagi, S.; Singh, R.; Carlton, J.M.; et al. Clinical and epidemiological characterization of severe Plasmodium vivax malaria in Gujarat, India. Virulence 2020, 11, 730-738. [CrossRef]

26. Arboleda, M.; Perez, M.F.; Fernandez, D.; Usuga, L.Y.; Meza, M. Clinical and laboratory profile of Plasmodium vivax malaria patients hospitalized in Apartado, Colombia. Biomedica 2012, 32 (Suppl. 1), 58-67. [CrossRef]

27. Arevalo-Herrera, M.; Lopez-Perez, M.; Medina, L.; Moreno, A.; Gutierrez, J.B.; Herrera, S. Clinical profile of Plasmodium falciparum and Plasmodium vivax infections in low and unstable malaria transmission settings of Colombia. Malar. J. 2015, 14, 154. [CrossRef] [PubMed]

28. Arévalo-Herrera, M.; Rengifo, L.; Lopez-Perez, M.; Arce-Plata, M.I.; García, J.; Herrera, S. Complicated malaria in children and adults from three settings of the Colombian Pacific Coast: A prospective study. PLoS ONE 2017, 12, e0185435. [CrossRef]

29. Barber, B.E.; William, T.; Grigg, M.J.; Parameswaran, U.; Piera, K.A.; Price, R.N.; Yeo, T.W.; Anstey, N.M. Effects of aging on parasite biomass, inflammation, endothelial activation, microvascular dysfunction and disease severity in Plasmodium knowlesi and Plasmodium falciparum malaria. J. Infect. Dis. 2017, 215, 1908-1917. [CrossRef]

30. Barber, B.E.; William, T.; Grigg, M.J.; Menon, J.; Auburn, S.; Marfurt, J.; Anstey, N.M.; Yeo, T.W. A prospective comparative study of knowlesi, falciparum, and vivax malaria in Sabah, Malaysia: High proportion with severe disease from Plasmodium knowlesi and Plasmodium vivax but no mortality with early referral and artesunate therapy. Clin. Infect. Dis. 2013, 56, 383-397. [CrossRef]

31. Dayanand, K.K.; Kishore, P.; Chandrashekar, V.; Achur, R.N.; Ghosh, S.K.; Kakkilaya, S.B.; Kumari, S.N.; Tiwari, S.; Boloor, A.; Devi, R.; et al. Malaria severity in Mangaluru city in the southwestern coastal region of India. Am. J. Trop. Med. Hyg. 2019, 100, 275-279. [CrossRef]

32. Douglas, N.M.; Pontororing, G.J.; Lampah, D.A.; Yeo, T.W.; Kenangalem, E.; Poespoprodjo, J.R.; Ralph, A.P.; Bangs, M.J.; Sugiarto, P.; Anstey, N.M.; et al. Mortality attributable to Plasmodium vivax malaria: A clinical audit from Papua, Indonesia. BMC Med. 2014, 12, 217. [CrossRef]

33. Gomes, L.T.; Alves-Junior, E.R.; Rodrigues-Jesus, C.; Nery, A.F.; Gasquez-Martin, T.O.; Fontes, C.J. Angiopoietin-2 and angiopoietin-2/angiopoietin-1 ratio as indicators of potential severity of Plasmodium vivax malaria in patients with thrombocytopenia. PLoS ONE 2014, 9, e109246.

34. Guedes, K.S.; Sanchez, B.A.M.; Gomes, L.T.; Fontes, C.J.F. Aspartate aminotransferase-to-platelet ratio index (APRI): A potential marker for diagnosis in patients at risk of severe malaria caused by Plasmodium vivax. PLoS ONE 2019, 14, e0224877. [CrossRef]

35. Gupta, P.; Guddattu, V.; Saravu, K. Characterization of platelet count and platelet indices and their potential role to predict severity in malaria. Pathog. Glob. Health 2019, 113, 86-93. [CrossRef]

36. Gupta, P.; Sharma, R.; Chandra, J.; Kumar, V.; Singh, R.; Pande, V.; Singh, V. Clinical manifestations and molecular mechanisms in the changing paradigm of vivax malaria in India. Infect. Genet. Evol. 2016, 39, 317-324. [CrossRef] [PubMed]

37. Jain, V.; Agrawal, A.; Singh, N. Malaria in a tertiary health care facility of Central India with special reference to severe vivax: Implications for malaria control. Pathog. Glob. Health 2013, 107, 299-304. [CrossRef]

38. Kumar, R.; Saravu, K. Severe vivax malaria: A prospective exploration at a tertiary healthcare centre in Southwestern India. Pathog. Glob. Health 2017, 111, 148-160. [CrossRef]

39. Kumari, M.; Ghildiyal, R. Clinical profile of Plasmodium vivax malaria in children and study of severity parameters in relation to mortality: A tertiary care centre perspective in Mumbai, India. Malar. Res. Treat. 2014, 2014, 765657. [CrossRef]

40. Kute, V.B.; Trivedi, H.L.; Vanikar, A.V.; Shah, P.R.; Gumber, M.R.; Patel, H.V.; Goswami, J.G.; Kanodia, K.V. Plasmodium vivax malaria-associated acute kidney injury, India, 2010-2011. Emerg. Infect. Dis. 2012, 18, 842-845. [CrossRef]

41. Lança, E.F.C.; Magalhães, B.M.L.; Vitor-Silva, S.; Siqueira, A.M.; Benzecry, S.G.; Alexandre, M.A.A.; O’Brien, C.; Bassat, Q.; Lacerda, M.V.G. Risk factors and characterization of Plasmodium vivax-associated admissions to pediatric intensive care units in the Brazilian Amazon. PLoS ONE 2012, 7, e35406. [CrossRef]

42. Manning, L.; Laman, M.; Law, I.; Bona, C.; Aipit, S.; Teine, D.; Warrell, J.; Rosanas-Urgell, A.; Lin, E.; Kiniboro, B.; et al. Features and prognosis of severe malaria caused by Plasmodium falciparum, plasmodium vivax and mixed plasmodium species in Papua New Guinean children. PLoS ONE 2011, 6, e29203. [CrossRef]

43. Mathews, S.E.; Bhagwati, M.M.; Agnihotri, V. Clinical spectrum of Plasmodium vivax infection, from benign to severe malaria: A tertiary care prospective study in adults from Delhi, India. Trop. Parasitol. 2019, 9, 88-92. [CrossRef]

44. O'Brien, A.T.; Ramirez, J.F.; Martinez, S.P. A descriptive study of 16 severe Plasmodium vivax cases from three municipalities of Colombia between 2009 and 2013. Malar. J. 2014, 13, 404. [CrossRef]

45. Park, S.Y.; Park, Y.S.; Park, Y.; Kwak, Y.G.; Song, J.E.; Lee, K.S.; Cho, S.H.; Lee, S.E.; Shin, H.I.; Yeom, J.S. Severe vivax malaria in the Republic of Korea during the period 2000 to 2016. Travel Med. Infect. Dis. 2019, 30, 108-113. [CrossRef]

46. Punnath, K.; Dayanand, K.K.; Chandrashekar, V.N.; Achur, R.N.; Kakkilaya, S.B.; Ghosh, S.K.; Mukhi, B.; Midya, V.; Kumari, S.N.; Gowda, D.C. Clinical features and haematological parameters among malaria patients in Mangaluru city area in the southwestern coastal region of India. Parasitol. Res. 2020, 119, 1043-1056. [CrossRef] 
47. Rajeshwar, K.K.B. Clinical profile of severe Plasmodium vivax malaria in a tertiary centre in J.J.M. Medical College, Davangere. Indian J. Basic Appl. Med. Res. 2015, 4, 133-139.

48. Raposo, C.C.; Santos, J.B.; Santos, G.M.; Goncalves Eda, G.; Silva, A.R. Plasmodium vivax malaria: Related factors to severity in the State of Maranhao, Brazil. Rev. Da Soc. Bras. De Med. Trop. 2013, 46, 67-72. [CrossRef]

49. Sharma, R.; Gohain, S.; Chandra, J.; Kumar, V.; Chopra, A.; Chatterjee, S.; Aneja, S.; Kumar Dutta, A. Plasmodium vivax malaria admissions and risk of mortality in a tertiary-care children's hospital in North India. Paediatr. Int. Child Health 2012, 32, 152-157. [CrossRef]

50. Singh, H.; Parakh, A.; Basu, S.; Rath, B. Plasmodium vivax malaria: Is it actually benign? J. Infect. Public Health 2011, 4, 91-95. [CrossRef]

51. Sud, R.; Singh, H.; Aggarwal, A.; Bhatnagar, M.K. Resurgence of complicated malaria associated with severe thrombocytopenia in a tertiary care hospital in Delhi. J. Assoc. Physicians India 2012, 60, 68.

52. Val, F.; Avalos, S.; Gomes, A.A.; Zerpa, J.E.A.; Fontecha, G.; Siqueira, A.M.; Bassat, Q.; Alecrim, M.G.C.; Monteiro, W.M.; Lacerda, M.V.G. Are respiratory complications of Plasmodium vivax malaria an underestimated problem? Malar. J. 2017, 16, 495. [CrossRef]

53. William, T.; Menon, J.; Rajahram, G.; Chan, L.; Ma, G.; Donaldson, S.; Khoo, S.; Fredrick, C.; Jelip, J.; Anstey, N.M.; et al. Severe Plasmodium knowlesi malaria in a tertiary care hospital, Sabah, Malaysia. Emerg. Infect. Dis. 2011, 17, 1248-1255. [CrossRef]

54. Willmann, M.; Ahmed, A.; Siner, A.; Wong, T.; Woon, L.C.; Singh, B.; Krishna, S.; Cox-Singh, J. Laboratory markers of disease severity in Plasmodium knowlesi infection: A case control study. Malar. J. 2012, 11, 363. [CrossRef]

55. Kotepui, M.; Phunphuech, B.; Phiwklam, N.; Chupeerach, C.; Duangmano, S. Effect of malarial infection on haematological parameters in population near Thailand-Myanmar border. Malar. J. 2014, 13, 218. [CrossRef]

56. Erhart, L.M.; Yingyuen, K.; Chuanak, N.; Buathong, N.; Laoboonchai, A.; Miller, R.S.; Meshnick, S.R.; Gasser, R.A.; Wongsrichanalai, C. Hematologic and clinical indices of malaria in a semi-immune population of western Thailand. Am. J. Trop. Med. Hyg. 2004, 70, 8-14. [CrossRef]

57. Lampah, D.A.; Yeo, T.W.; Malloy, M.; Kenangalem, E.; Douglas, N.M.; Ronaldo, D.; Sugiarto, P.; Simpson, J.A.; Poespoprodjo, J.R.; Anstey, N.M.; et al. Severe malarial thrombocytopenia: A risk factor for mortality in Papua, Indonesia. J. Infect. Dis. 2015, 211, 623-634. [CrossRef]

58. Gerardin, P.; Rogier, C.; Ka, A.S.; Jouvencel, P.; Brousse, V.; Imbert, P. Prognostic value of thrombocytopenia in African children with falciparum malaria. Am. J. Trop. Med. Hyg. 2002, 66, 686-691. [CrossRef]

59. Pain, A.; Ferguson, D.J.; Kai, O.; Urban, B.C.; Lowe, B.; Marsh, K.; Roberts, D.J. Platelet-mediated clumping of Plasmodium falciparum-infected erythrocytes is a common adhesive phenotype and is associated with severe malaria. Proc. Natl. Acad. Sci. USA 2001, 98, 1805-1810. [CrossRef]

60. WHO. Severe malaria. Trop. Med. Int. Health 2014, 19, 7-131. [CrossRef]

61. Kotepui, M.; Piwkham, D.; PhunPhuech, B.; Phiwklam, N.; Chupeerach, C.; Duangmano, S. Effects of malaria parasite density on blood cell parameters. PLOS ONE 2015, 10, e0121057. [CrossRef]

62. Tagny, C.T.; Mbanya, D.; Tapko, J.B.; Lefrere, J.J. Blood safety in Sub-Saharan Africa: A multi-factorial problem. Transfusion 2008, 48, 1256-1261. [CrossRef]

63. Looareesuwan, S.; Davis, J.G.; Allen, D.L.; Lee, S.H.; Bunnag, D.; White, N.J. Thrombocytopenia in malaria. Southeast Asian J. Trop. Med. Public Health 1992, 23, 44-50. [PubMed]

64. Bottieau, E.; Clerinx, J.; Van Den Enden, E.; Van Esbroeck, M.; Colebunders, R.; Van Gompel, A.; Van Den Ende, J. Imported non-Plasmodium falciparum malaria: A five-year prospective study in a European referral center. Am. J. Trop. Med. Hyg. 2006, 75, 133-138. [CrossRef] [PubMed]

65. Langford, S.; Douglas, N.M.; Lampah, D.A.; Simpson, J.A.; Kenangalem, E.; Sugiarto, P.; Anstey, N.M.; Poespoprodjo, J.R.; Price, R.N. Plasmodium malariae Infection Associated with a High Burden of Anemia: A hospital-based surveillance study. PLoS Negl. Trop. Dis. 2015, 9, e0004195. [CrossRef]

66. Rojo-Marcos, G.; Rubio-Muñoz, J.M.; Ramírez-Olivencia, G.; García-Bujalance, S.; Elcuaz-Romano, R.; Díaz-Menéndez, M.; Calderón, M.; García-Bermejo, I.; Ruiz-Giardín, J.M.; Merino-Fernández, F.J.; et al. Comparison of imported Plasmodium ovale curtisi and P. ovale wallikeri infections among patients in Spain, 2005-2011. Emerg. Infect. Dis. 2014, 20, 409-416. [CrossRef] [PubMed]

67. Turner, G.D.; Morrison, H.; Jones, M.; Davis, T.M.; Looareesuwan, S.; Buley, I.D.; Gatter, K.C.; Newbold, C.I.; Pukritayakamee, S.; Nagachinta, B.; et al. An immunohistochemical study of the pathology of fatal malaria. Evidence for widespread endothelial activation and a potential role for intercellular adhesion molecule-1 in cerebral sequestration. Am. J. Pathol. 1994, 145, 1057-1069.

68. Sadler, J.E. Biochemistry and genetics of von Willebrand factor. Ann. Rev. Biochem. 1998, 67, 395-424. [CrossRef] [PubMed]

69. Löwenberg, E.C.; Charunwatthana, P.; Cohen, S.; van den Born, B.J.; Meijers, J.C.; Yunus, E.B.; Hassan, M.B.; Hoque, G.; Maude, R.J.; Nuchsongsin, F.; et al. Severe malaria is associated with a deficiency of von Willebrand factor cleaving protease, ADAMTS13. Thromb. Haemost. 2010, 103, 181-187. [CrossRef] [PubMed]

70. Morrell, C.N.; Aggrey, A.A.; Chapman, L.M.; Modjeski, K.L. Emerging roles for platelets as immune and inflammatory cells. Blood 2014, 123, 2759-2767. [CrossRef]

71. Boutlis, C.S.; Yeo, T.W.; Anstey, N.M. Malaria tolerance-for whom the cell tolls? Trends Parasitol. 2006, 22, 371-377. [CrossRef] 
72. Yeo, T.W.; Lampah, D.A.; Gitawati, R.; Tjitra, E.; Kenangalem, E.; McNeil, Y.R.; Darcy, C.J.; Granger, D.L.; Weinberg, J.B.; Lopansri, B.K.; et al. Impaired nitric oxide bioavailability and L-arginine reversible endothelial dysfunction in adults with falciparum malaria. J. Exp. Med. 2007, 204, 2693-2704. [CrossRef] [PubMed]

73. Kong, T.F.; Ye, W.; Peng, W.K.; Hou, H.W.; Preiser, P.R.; Nguyen, N.T.; Han, J. Enhancing malaria diagnosis through microfluidic cell enrichment and magnetic resonance relaxometry detection. Sci. Rep. 2015, 5, 11425. [CrossRef]

74. Di Gregorio, E.; Ferrauto, G.; Schwarzer, E.; Gianolio, E.; Valente, E.; Ulliers, D.; Aime, S.; Skorokhod, O. Relaxometric studies of erythrocyte suspensions infected by Plasmodium falciparum: A tool for staging infection and testing anti-malarial drugs. Magn. Reson. Med. 2020, 84, 3366-3378. [CrossRef]

75. Peng, W.K.; Kong, T.F.; Ng, C.S.; Chen, L.; Huang, Y.; Bhagat, A.A.S.; Nguyen, N.T.; Preiser, P.R.; Han, J. Micromagnetic resonance relaxometry for rapid label-free malaria diagnosis. Nat. Med. 2014, 20, 1069-1073. [CrossRef]

76. Gupta, M.; Singh, K.; Lobiyal, D.K.; Safvan, C.P.; Sahu, B.K.; Yadav, P.; Singh, S. A sensitive on-chip probe-based portable nuclear magnetic resonance for detecting low parasitaemia Plasmodium falciparum in human blood. Devices Sens. 2020, 3, e10098. [CrossRef]

77. Arndt, L.; Koleala, T.; Orbán, Á.; Ibam, C.; Lufele, E.; Timinao, L.; Lorry, L.; Butykai, Á.; Kaman, P.; Molnár, A.P.; et al. Magneto-optical diagnosis of symptomatic malaria in Papua New Guinea. Nat. Commun. 2021, 12, 969. [CrossRef]

78. Yamamoto, T.; Hashimoto, M.; Nagatomi, K.; Nogami, T.; Sofue, Y.; Hayashi, T.; Ido, Y.; Yatsushiro, S.; Abe, K.; Kajimoto, K. Field Evaluation of a Quantitative, and Rapid Malaria Diagnostic System Using a Fluorescent Blue-Ray Optical Device. 2019. Available online: https: / / www.biorxiv.org/content/10.1101/721076v1 (accessed on 23 October 2021). 